\title{
Simplified visual aid to detect early CT findings in COVID-19 pneumonia for non-radiologists
}

\author{
Fikri M. Abu-Zidan ${ }^{1}\left[\right.$ ] Taleb M. Almansoori ${ }^{2}$
}

Received: 14 April 2020 / Accepted: 17 July 2020 / Published online: 24 July 2020

○) Springer-Verlag GmbH Germany, part of Springer Nature 2020

\section{Dear Editor,}

Real-time transcription polymerase chain reaction (RT-PCR) assay is the gold standard for diagnosing COVID-19. Nevertheless, it may be negative in the early 3-8 days of the active disease before becoming positive, or it may not be available [1, 2].

Early CT scan findings are very sensitive in detecting COVID-19 disease. Only five out of 167 (3\%) patients who had these findings did not have the disease [2]. Accordingly, it is very important for acute-care physicians to be familiar with these findings so that they can independently read the CT scan images of COVID-19 pneumonia patients when radiologists are overwhelmed or not available. This will help isolating highly suspected patients depending on the CT scan findings and not to be mixed with other non-COVID-19 surgical patients before getting the RT-PCR results.

After critically reading the recently published CT scan findings of COVID-19 [1-5], we noticed that the used terminology is difficult for non-radiologist to understand. We tried to simplify the reported radiological findings for the nonradiologists using a simple illustration so that they can be encouraged to read the actual CT scan images independently.

The most common, important, and easy image finding that should be obvious for acute-care physicians with limited experience in reading a chest $\mathrm{CT}$ scan is the peripheral ground-glass opacities (GGO). GGO is an area of increased attenuation with hazy margin which does not affect the

Fikri M. Abu-Zidan

fabuzidan@uaeu.ac.ae

Taleb M. Almansoori

taleb.almansoor@uaeu.ac.ae

1 Department of Surgery, College of Medicine and Health Sciences, UAE University, Al-Ain, United Arab Emirates

2 Department of Radiology, College of Medicine and Health Sciences, UAE University, Al-Ain, United Arab Emirates

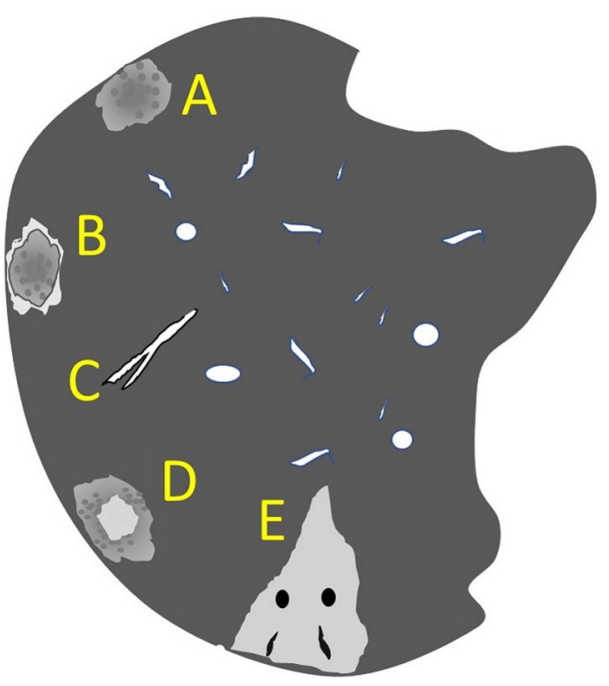

Fig. 1 A simplified illustration demonstrating the early CT findings of COVID-19 pneumonia which include: a ground-glass opacity (GGO), b reversed halo sign, $\mathbf{c}$ vascular dilatation, $\mathbf{d}$ combined GGO and consolidation, and finally e advanced consolidation. (Illustrated by Professor Fikri Abu-Zidan)

vascular or bronchial margin (Figs. 1 and 2a). This finding should be sufficient for non-radiologists to suspect COVID-19.

Other useful radiological findings for the acute-care physicians who are experienced in reading CT scans of the chest include the reversed halo sign (B), vascular dilatation (C), combined GGO and consolidation (D), and finally advanced consolidation (E) [1-5] (Figs. 1 and 2b-e). Consolidation is an area of opacification that may surround the GGO (reversed halo sign) or within the GGO, while advanced consolidation is an area of opacification that does not include GGO [1-5].

The percentages of other useful radiological findings in chest CT scans are as follows: the halo sign (64\%), vascular dilation (68-80\%), GGO with consolidation (41-64\%), and 


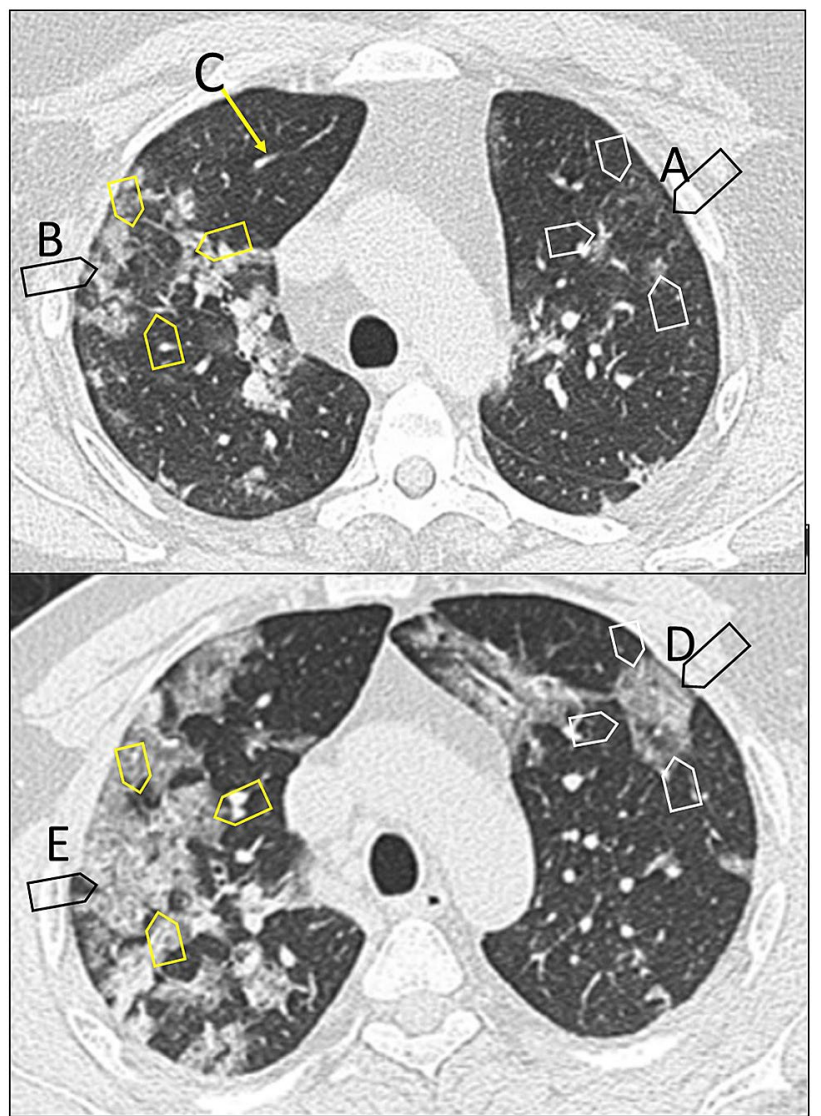

Fig. 2 Chest CT scan images of two patients proven to have COVID19 disease demonstrate the early CT findings of COVID-19 pneumonia which include: a ground-glass opacity (GGO), b reversed halo sign, $\mathbf{c}$ vascular dilatation, $\mathbf{d}$ combined GGO and consolidation, and finally $\mathbf{e}$ advanced consolidation

pure consolidation $(6-41 \%)[4,5]$. All the above five findings are located peripherally in the sub-pleural regions of the lungs and are present in $96 \%$ of patients [1].
The Radiological Society of North America has built an excellent website for CVOID-19 chest pneumonia images (https://cases.rsna.org/coronavirus) by which non-radiologists can familiarize themselves with these important findings within a short time by reading actual CT images. COVID-19 pandemic is not yet over and may stay with us for long time. We hope that this illustration and the CT scan images will encourage acute-care physicians to read chest CT scans independently when needed in this difficult time.

\section{Compliance with ethical standards}

Conflict of interest Fikri Abu-Zidan and Taleb M. Almansoori declare that they have no conflict of interest regarding this letter.

Compliance with ethical requirements Radiological images do not have any identifier or clinical data. The patients signed a consent to use their images for research.

\section{References}

1. Li Y, Xia L. Coronavirus disease 2019 (COVID-19): role of chest $\mathrm{CT}$ in diagnosis and management. AJR Am J Roentgenol. 2020;214:1280-6.

2. Xie X, Zhong Z, Zhao W, Zheng C, Wang F, Liu J. Chest CT for typical 2019-nCoV pneumonia: relationship to negative RT-PCR testing. Radiology. 2020;12:200343. https://doi.org/10.1148/radio 1.2020200343 (Epub ahead of print).

3. Zu ZY, Jiang MD, Xu PP, et al. Coronavirus disease 2019 (COVID-19): a perspective from china. Radiology. 2020. https:// doi.org/10.1148/radiol.2020200490(Epub ahead of print).

4. Han R, Huang L, Jiang H, Dong J, Peng H, Zhang D. Early clinical and CT manifestations of coronavirus disease 2019 (COVID19) pneumonia. AJR Am J Roentgenol. 2020;17:1-6. https://doi. org/10.2214/AJR.20.22961(Epub ahead of print).

5. Zhao W, Zhong Z, Xie X, Yu Q, Liu J. Relation between chest CT findings and clinical conditions of coronavirus disease (COVID19) pneumonia: a multicenter study. AJR Am J Roentgenol. 2020;214:1072-7. 